\title{
Percutaneous closure of atrial septal defect in patients under 15 kilograms using balloon assisted technique. Case report
}

\author{
Cierre percutáneo de comunicación interauricular en paciente con peso menor de 15 \\ kilogramos utilizando técnica asistida con balón. Reporte de un caso
}

\author{
Guillermo Aristizabal-Villa ${ }^{1, *}$, Alexis Plata-Marriaga ${ }^{2}$ and Jorge Escobar-Modesto ${ }^{3}$ \\ ${ }^{1}$ Department of Pediatric Cardiology and Hemodynamics, Clínica de la Costa, Barranquilla; ${ }^{2}$ Department of Research, Clínica Madre Bernarda, \\ Cartagena; ${ }^{3}$ Department of Pediatrics, Clínica de la Costa, Barranquilla. Colombia
}

\section{Case presentation}

Three-year-old girl weighing $14 \mathrm{~kg}$ who had a history of community-acquired pneumonia episodes and atrial septal defect. On physical examination, grade II/IV systolic murmur on pulmonary area and splitting of second heart sound was found on auscultation. Chest X-ray showed a cardiothoracic index of 0.6 with pulmonary hyperflow. On electrocardiogram, right axis deviation and incomplete right bundle branch block were observed. Transthoracic echocardiogram revealed an ostium secundum atrial septal defect of $14 \mathrm{~mm}$ in diameter with left-to-right shunt, and right ventricular and pulmonary artery significant dilatation. The rims of the defect were evaluated, with a top rim of $10 \mathrm{~mm}$, bottom rim of $8 \mathrm{~mm}$ and postero-inferior rim of $9 \mathrm{~mm}$ being found, with absence of anterior rim (relative to the aorta). Percutaneous closure of the defect was decided due to the presence of data consistent with hemodynamic impact. During hemodynamics assessment, with oxygen at $21 \%$, mean pulmonary artery pressure of $18 \mathrm{mmHg}$, right atrium oxygen saturation oscillation, Qp/Qs 3.9/1 and indexed pulmonary vascular resistance of 0.8 Wood units per square meter of body surface area were found. No other shunts were found on angiographic diagnosis. Aided by transesophageal echocardiogram, interatrial septum was evaluated, with an ostium secundum-type defect of $14 \mathrm{~mm}$ in diameter being found, with anterior (aortic) rim absence being corroborated. Following heparin administration (100 U/kg) and activated coagulation time registration (> $250 \mathrm{~s}$ ), on postero-anterior angiographic projection, closing the defect with an 18-mm Occlutech Figulla Flex II device was attempted. Dislocation of the device's left disk was observed over the aorta, towards the right atrium, due to the lack of support by the anterior rim of the defect. Supporting the device with the balloon-assisted technique was then considered. Another left femoral venous access was obtained and the guidewire was positioned in the left superior pulmonary vein, advancing the $25-\mathrm{mm}$ sizing balloon (Occlutech Sizing Balloon), positioning it through the septal defect and partially inflating it. The left disk was then released into the left atrium (Fig. 1), with the device and the releasing system being retracted together towards the interatrial septum, and the right disk being released into the right atrium, without left disk dislocation being observed. The balloon was deflated and removed, and the device was released with no further complications (Fig. 2). The patient showed no electrocardiographic alterations during the intervention. Procedure time was 60 minutes. No additional heparin dose was required. At 24 hours of the
Correspondence:

*Guillermo Aristizabal-Villa

E-mail: Guillermo.aristizabal68@gmail.com license (http://creativecommons.org/licenses/by-nc-nd/4.0/).

Date of reception: $28-06-2020$

Date of acceptance: $21-08-2020$

DOI: 10.24875/ACME.M21000207
Available online: 19-07-2021

Arch Cardiol Mex (Eng). 2021;91(2):229-231

www.archivoscardiologia.com 

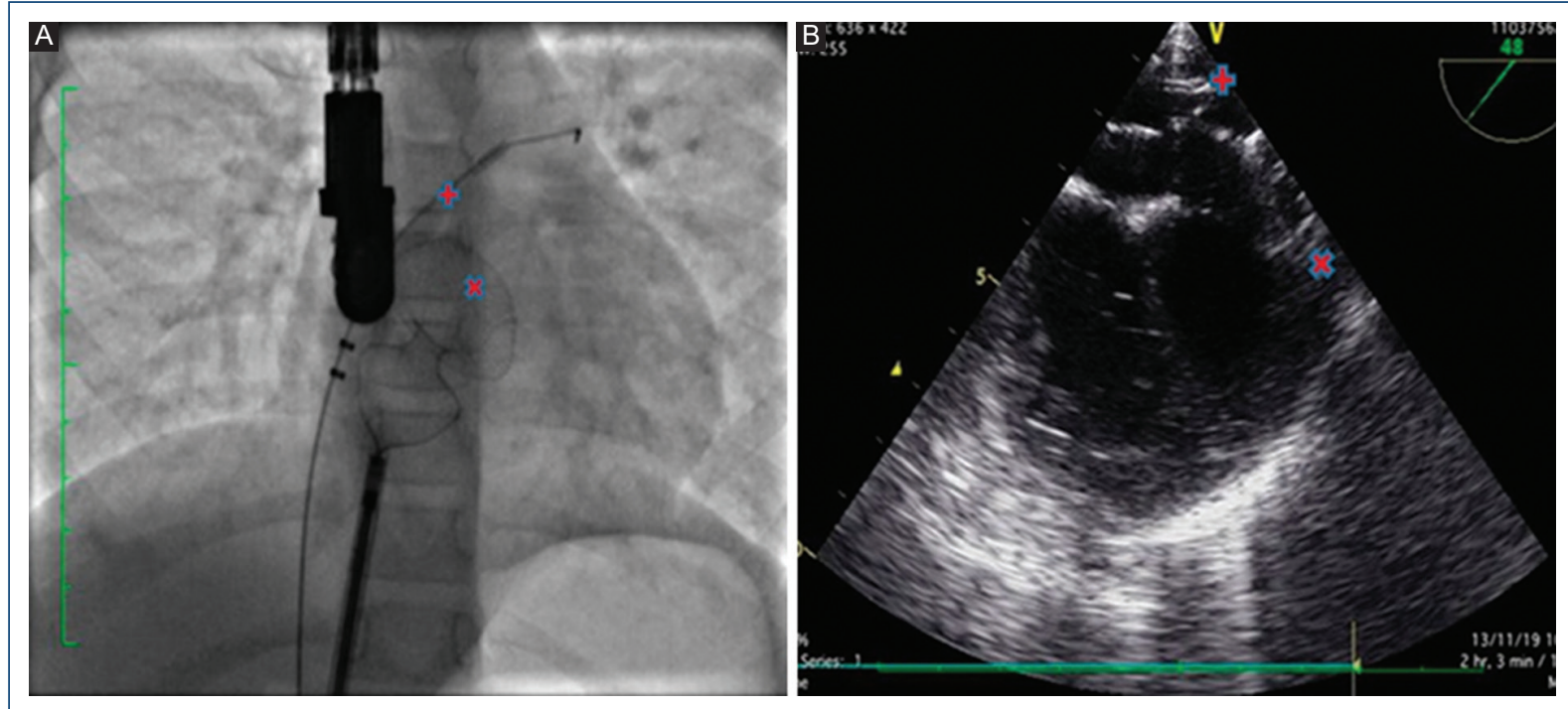

Figure 1. A: Fluoroscopic image. B: Simultaneous transesophageal echocardiography. The atrial defect occlusive device is observed unfolding (x). The sizing balloon is used partially inflated (+) to give support to the device's left disk, with its position on the left atrium being maintained and thus avoiding its displacement or dislocation towards the right atrium.
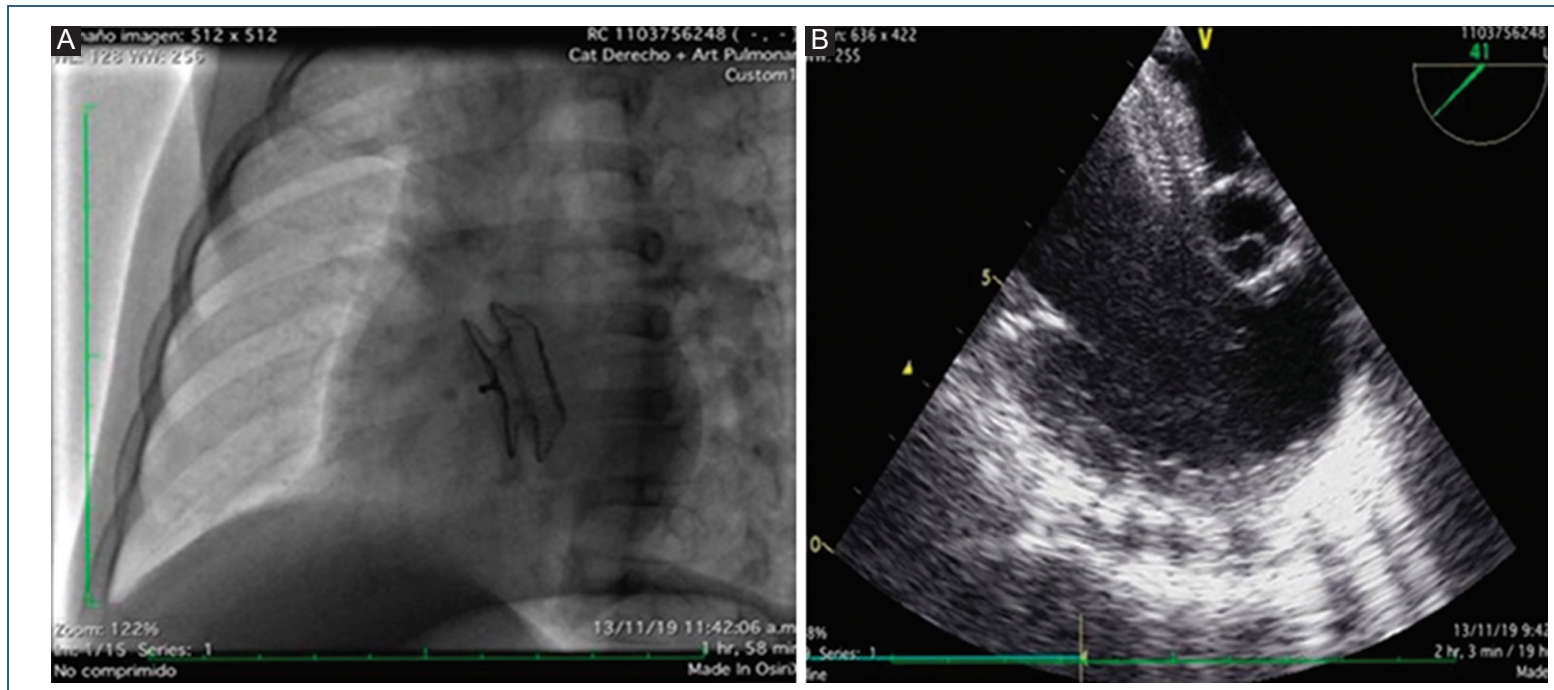

Figure 2. A: Fluoroscopic image. B: Simultaneous transesophageal echocardiography. The atrial septal defect occlusive device released with adequate implantation on the interatrial septum is observed (B), with optimal separation of the disks (A).

procedure, transthoracic echocardiogram was performed, with the device being found in the correct position, without displacement and without pericardial effusion. The patient was discharged at 24 hours on treatment with acetylsalicylic acid and clopidogrel for 6 and 2 months, respectively. No electrocardiographic or echocardiographic alterations were found at one month and at 6 -month follow-up evaluation.
Most children with atrial septal defect are asymptomatic or exhibit mild symptoms, and thus percutaneous closure tends to be postponed until 4 years of age or until reaching more than $15 \mathrm{~kg}$ of weight, due to the high rate of spontaneous closure of defects smaller than $8 \mathrm{~mm}$ within the first years of life $^{1,2}$. Some patients with a large left-to-right shunt experience failure to thrive, repeated lower respiratory tract 
infections or asthma attack episodes with multiple hospitalizations, and in this cases, closure of the defect would be indicated despite weight and age, mainly in those with a history of prematurity or chromosomal abnormalities. Atrial septal defects in this group of patients are associated with a smaller left atrial cavity, which results in higher difficulty for positioning the left disk, thus generating misalignment with interatrial septum and dislocation of the device through the defect. Consequently, the usual closure technique tends to fail in most these cases. Sharma et al. ${ }^{2}$ published a study with 45 pediatric patients, with a mean of 25 months of age and average weight of $8.9 \mathrm{~kg}$, in whom the usual release technique was effective only in 15 patients, with special release maneuvers being required in 28 patients; the most commonly used was the release into the left superior pulmonary vein, with 18 cases, followed by the balloon-assisted technique in six cases, release into the right superior pulmonary vein in three cases and release into the left appendage in one case. The balloon-assisted technique has the disadvantage of requiring another venous vascular access, an additional operator to hold the balloon, and longer procedure and hemostasis time $^{3}$. Septal defect closure efficacy has been demonstrated in low-weight patients, with improvement in symptoms and weight for age in long-term follow-up, as well as regression in echocardiographic data of right ventricular volume overload $^{4}$. As for safety of the procedure, the risk of major and minor complications is comparable to that of older and heavier patients, taking into account that younger patients with low weight have comorbidity associated with prolonged hospital stay ${ }^{5}$.

As conclusions, atrial septal defects percutaneous closure in patients with low weight or age has specific indications, such as failure to thrive, recurrent respiratory infections associated with prematurity or chromosomal abnormalities. The usual percutaneous closure technique in this type of patients is unsuccessful in a high percentage, given that the smaller size of the left atrium does not allow the device to be positioned on the interatrial septum. Special release maneuvers must be implemented, by means of which closure of the defect can be safely and efficaciously be carried out

\section{Conflicts of interest}

None.

\section{Funding}

This research has not received any specific grant from public, commercial or non-profit sector agencies.

\section{Ethical disclosures}

Protection of human and animal subjects. The authors declare that no experiments have been performed on humans or animals for this research.

Confidentiality of data. The authors declare that they have followed the protocols of their work center on the publication of patient data.

Right to privacy and informed consent. The authors declare that no patient data appear in this article.

\section{References}

1. Vogel M, Berger F, Dähnert I, Ewert P, Lange PE. Treatment of atrial septal defects in symptomatic children aged less than 2 years of age using the Amplatzer septal occluder. Cardiol Young. 2000;10:534-7.

2. Sharma B, Pinto R, Dalvi B. Transcatheter closure of atrial septal defect in symptomatic children weighing $\leq 10 \mathrm{~kg}$ : addressing unanswered issues from a decade of experience. Ann Pediatr Cardiol 2020;13:4-10.

3. Dalvi B. Balloon assisted technique for closure of large atrial septal defects. Images Paediatr Cardiol. 2008;10:5-9.

4. Wyss Y, Quandt D, Weber R, Stiasny B, Weber B, Knirsch W, et al. Interventional closure of secundum type atrial septal defects in infants less than 10 kilograms: indications and procedural outcome. J Interv Cardiol. 2016;29:646-53.

5. Tangho G, Odermarsky M, Naumburg E, Liuba P. Early complications after percutaneous closure of atrial septal defect in infants with procedural weight less than $15 \mathrm{~kg}$. Pediatr Cardiol. 2017;38:25563. 